'Departamento de Radiología, Clínica Las Condes. Santiago, Chile. Profesor Universidad de Chile. Santiago, Chile. ${ }^{2}$ Departamento de Hematooncología y Jefe de Banco de Sangre, Clínica Las Condes, Santiago, Chile.

${ }^{3}$ Alumno de Medicina, Facultad de Medicina, Universidad de Chile. Santiago, Chile. ${ }^{4}$ Becaria especialidad de Radiología, Universidad de Santiago de Chile. Santiago, Chile.

${ }^{5}$ Médico Nuclear en estadía de observación en Unidad de PET/ $\mathrm{CT}$, Clínica Las Condes. Santiago, Chile.

Trabajo no recibió financiamiento. Los autores declaran no tener conflictos de interés.

Recibido el 28 de mayo de 2020, aceptado el 4 de noviembre de

Correspondencia a: Dr. David Ladrón de Guevara H. Servicio de Radiología, Clínica Las Condes. Santiago, Chile. dlg@clc.cl

\section{Valor pronóstico del PET/CT interino en Linfoma no Hodgkin en comparación con factores pronósticos clásicos (IPI) y de composición corporal (sarcopenia/lipopenia)}

\author{
DAVID LADRÓN DE GUEVARA ${ }^{1}$, SEBASTIÁN BERNARD ${ }^{3}$, \\ SUSANA MANHOOD ${ }^{3}$, SOPHIA MELANI ${ }^{4}$, FERNANDO YEROVI ${ }^{5}$, \\ MARÍA DE LOS ÁNGELES RODRÍGUEZ ${ }^{2}$

\section{Prognostic value of interim PET/CT in non-hodgkin lymphoma}

Background: The prognosis of Non-Hodgkin Lymphoma (NHL) depends on the type of lymphoma, the extension of the disease and the response to therapy. Aim: To evaluate the prognostic value of pretreatment and interim PET/ CT compared to classic prognosis factors and body composition measurement (sarcopenia, adipopenia) in patients with recently diagnosed NHL. Material and Methods: Patients with recently diagnosed NHL who had staging ${ }^{18} F-F D G$ PET/CT performed between December 2008 and August 2018 were selected. Age, gender, weight, height, B symptoms, laboratory tests, pathology, staging PET/ CT findings (Ann Arbor, number of nodal groups and extranodal sites involved, Bulky, maximum standardized uptake value (SUVmax), metabolic tumor volume (MTV), and total lesion glycolysis), Computed Tomography findings (psoas muscle mass index, psoas area, psoas density, subcutaneous fat index (all of them at L3 level), and Deauville score (Lugano Criteria) were recorded. The prognostic value of each of these factors was assessed using Cox multivariable regressions. Results: Of 138 NHL studied patients (median 61 y, 15-87 y, 60.4\% men), 31 of them died due to the disease. The median follow-up was 39 months (1-115 months). The strongest prognostic factors were: $B$ symptoms $(p<0.01)$, anemia ( $p<0.01)$, hypoalbuminemia ( $p: 0.01)$, sarcopenia $(p<0.01)$, adipopenia $(p<0.01)$, number of node groups involved $(p<0.01), M T V(p<0.01)$, and a bad response in interim PET/CT $(p<0.01)$. In a comparative Cox multivariable analysis, interim PET/CT was the independent variable with the highest significance $(p<0.01)$. Conclusions: Early treatment response assessed by interim PET/CT is the strongest prognostic factor in NHL patients.

(Rev Med Chile 2020; 148: 1558-1567)

Key words: Lymphoma, Non-Hodgkin; Multidetector Computed Tomography; Prognosis; Positron-Emission Tomography; Survival.

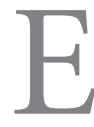

1 Linfoma no Hodgkin (LNH) corresponde a un grupo heterogéneo de neoplasias originadas de células linfocitarias T, Natural Killer o precursores maduros ${ }^{1}$, con un amplio rango de presentaciones histológicas y manifestaciones clínica². Su incidencia anual en Chile es de apro- ximadamente 5,6 casos por 100.000 habitantes ${ }^{1}$, con tendencia al aumento en nuestro país y en el mundo ${ }^{2}$. La incidencia en Estados Unidos para el período 2012-2017 fue de 19,6 casos por 100.000 habitantes ${ }^{3}$.

Actualmente, el 40-50\% de los LNH pueden 
llegar a curarse según literatura internacional ${ }^{4}$. En nuestro país, la mortalidad por LNH es de 3-4 por 100.000 habitantes $^{1}$, con una sobrevida global de $35 \%$ a 5 años ${ }^{1}$.

El pronóstico depende fuertemente del tipo de LNH, la extensión de la enfermedad al momento del diagnóstico y la respuesta a terapia ${ }^{5}$.

Existen diversos subtipos de $\mathrm{LNH}^{6}$, cada uno de ellos con diferente pronóstico. La sobrevida a 5 años del LNH difuso de células grandes (DCG) es de $63 \%$, y del linfoma Folicular (LF) es de $88 \%{ }^{5}$. Aquellos LNH de curso más lento, los llamados linfomas indolentes, pueden tener sobrevidas aún mayores ${ }^{7}$. Estos corresponden entre otros a la leucemia linfática crónica/linfocítico de células pequeñas $^{8}$, de zona marginal ${ }^{5,8}$, folicular ${ }^{2,5,8}$ y otros menos frecuentes ${ }^{2}$.

Una herramienta aceptada transversalmente en la evaluación pronóstica del linfoma es el Índice Pronóstico Internacional (IPI) ${ }^{2}$, que depende de 5 factores: Edad ( $\leq 60$ vs $>60$ años), etapa de avance de la enfermedad (Ann Arbor I-II vs III-IV), existencia de compromiso extranodal, estado general o performance status, y nivel de láctico deshidrogenasa sanguíneo $(\mathrm{LDH})^{9}$. El IPI es de utilidad en casi todos los LNH, con ciertas variantes introducidas para algunos subtipos histológicos como el LF, donde se agrega la hemoglobina (valor de corte de $12 \mathrm{~g} / \mathrm{dl}$ ) como otro factor a considerar ${ }^{2,9}$.

La extensión de la enfermedad al momento del diagnóstico es de gran importancia en determinar pronóstico. La enfermedad localizada en una sola área nodal o extranodal, correspondiente a etapa Ann Arbor I, tiene una sobrevida de $95 \%$ a 5 años en LF. La etapa II que comprometa 2 o más grupos nodales a un mismo lado del diafragma presenta sobrevidas de $90 \%$ a 5 años en LF. Por otro lado, una enfermedad diseminada a órganos o ganglios a ambos lados del diafragma (Etapa III-IV) presenta sobrevidas de $84 \%$ a 5 años en LF 5 .

La carga tumoral es sin lugar a dudas información pronóstica valiosa en pacientes con $\mathrm{LNH}$ recientemente diagnosticado ${ }^{10}$, y puede ser deducida parcialmente a través de la clasificación Ann Arbor, y marcadores tumorales como la $\mathrm{LDH}^{10}$. Otra manera eficiente de estimar la masa tumoral total es mediante estudios de imagen funcionales de cuerpo completo, como la antigua cintigrafía con Galio- $67^{11}$ y el más reciente PET/CT (Positron Emission Tomography/Computed Tomography) con F18-Fluorodeoxiglucosa (F18-FDG) ${ }^{9}$. Éste último, permite además cuantificarla de manera precisa a través de softwares que detecten y midan la extensión del compromiso nodal y extranodal, expresándolo mediante índices como el volumen metabólico total (VMT). Otros índices semi-cuantitativos empleados rutinariamente son el SUV (Standarized uptake value), que estima la intensidad de captación, y la masa tumoral glicolítica (MTG), que es el producto de ambos, es decir combina intensidad y extensión. Dichos índices han demostrado valor pronóstico en distintos tipos de LNH como el primario mediastínico ${ }^{12}$, Linfoma del manto ${ }^{13}$, y otros $\mathrm{LNH}^{14}$.

Particularmente, la intensidad de captación de F18-FDG objetivada mediante SUV ha mostrado una relación directa con la agresividad del $\mathrm{LNH}$, con mayor actividad metabólica en linfomas de alto grado, y menor captación en linfomas indolentes ${ }^{15,16}$.

Las relaciones de composición corporal, específicamente la proporción de grasa y masa muscular, han sido propuestas como un nuevo factor pronóstico en pacientes con cáncer, incluyendo series de pacientes con LNH. La disminución de la masa muscular (sarcopenia) y de la masa adiposa (lipopenia) se han asociado a mal pronóstico en LNH DCG en algunas series ${ }^{17-19}$. Sin embargo, esta relación no ha sido demostrada para otros tipos de LNH.

Por último, la respuesta a terapia es de gran importancia pronóstica, y puede ser estimada clínicamente, por imágenes o por histopatología. El PET/CT con F18-FDG realizado interciclos de quimioterapia (PET/CT interino) es la manera más utilizada en la actualidad para evaluar respuesta a terapia en $\mathrm{LNH}_{\text {agresivos }}$. Su valor pronóstico de sobrevida ha sido demostrado largamente tanto en linfoma de Hodgkin como en $\mathrm{LNH}^{20}$.

El objetivo de este estudio es evaluar la utilidad pronóstica de sobrevida del PET/CT interino y compararlo a factores clásicos como el IPI, y a medidas de composición corporal (sarcopenia, lipopenia), en una población local de pacientes con LNH recientemente diagnosticado.

\section{Pacientes y Método}

Desde la base de datos de PET/CT de nuestra institución, se seleccionaron aquellos pacientes con $\mathrm{LNH}$ recientemente diagnosticado que se 
efectuaron PET/CT F18-FDG de cuerpo completo de etapificación, entre diciembre de 2008 y agosto de 2018. Aquellos pacientes que hubieran iniciado tratamiento de cualquier tipo fueron excluidos.

Se rescató el estudio histológico de todos los pacientes, determinando subtipo histológico de cada LNH. Los casos de histología compleja fueron consensuados entre dos patólogos expertos, y paralelamente enviados a un centro de referencia para su análisis (CDM Fletcher, M.D., Brigham and Women's Hospital). Los diagnósticos histológicos se basaron en los criterios WHO de clasificación de las neoplasias linfoides 6 .

Se registró la siguiente información en cada paciente: 1) aspectos clínicos: edad, sexo, peso, talla, índice de masa corporal (IMC), presencia de síntomas B (fiebre, sudoración, baja de peso); 2) exámenes de laboratorio: niveles sanguíneos de albúmina, $\beta 2$-microglobulina, hemoglobina, LDH, VHS); 3) hallazgos del PET/CT de etapificación: estadio Ann Arbor, $\mathrm{n}^{\circ}$ de grupos ganglionares comprometidos, $\mathrm{n}^{\circ}$ órganos extranodales, existencia de Bulky, SUV maximun (SUVmax) de la lesión dominante, VMT, MTG; 4) hallazgos de la Tomografía Computada no contrastada: índice muscular del psoas, área del psoas, densidad del psoas, e índice de grasa subcutánea, todos a nivel L3; 5) características histológicas: subtipo de LNH,
Ki67; y 6) respuesta al tratamiento medido con PET/CT interino post $2^{\circ}$ o $3^{\circ}$ ciclo de quimioterapia según graduación Deauville 9 . Los casos con PET/CT interino realizados post $4^{\circ}$ o $5^{\circ}$ ciclo fueron excluidos.

Para calcular el SUVmax, SUVpromedio, VMT y MTG se dibujó un área de interés automática 3D alrededor de las lesiones, excluyendo estructuras adyacentes con alta actividad fisiológica como el cerebro, corazón, y vías urinarias ${ }^{21}$. El VMT correspondió a la masa tumoral total expresado en cc, y se calculó mediante software oncológico propio del equipo (Figura 1). La MTG correspondió al VMT x SUVpromedio ${ }^{22}$.

El índice muscular del psoas (IMP) correspondió al promedio del área del psoas izquierdo y derecho medida manualmente en un corte axial de TC a nivel L3 (Figura 2), dividido por la estatura al cuadrado según la fórmula IMP = $\mathrm{cm}^{2} / \mathrm{m}^{223}$. Debido a que las mujeres presentan un IMP fisiológicamente menor a los hombres ${ }^{24}$, se normalizó su valor al de éstos para poder calcular el IMP de manera conjunta en los cálculos estadísticos, y expresarlos en la misma curva de sobrevida. La densidad del psoas se midió mediante área de interés en ambos psoas, y correspondió al promedio de ambos expresados como coeficiente de atenuación en unidades Hounsfield ${ }^{25}$. El índice

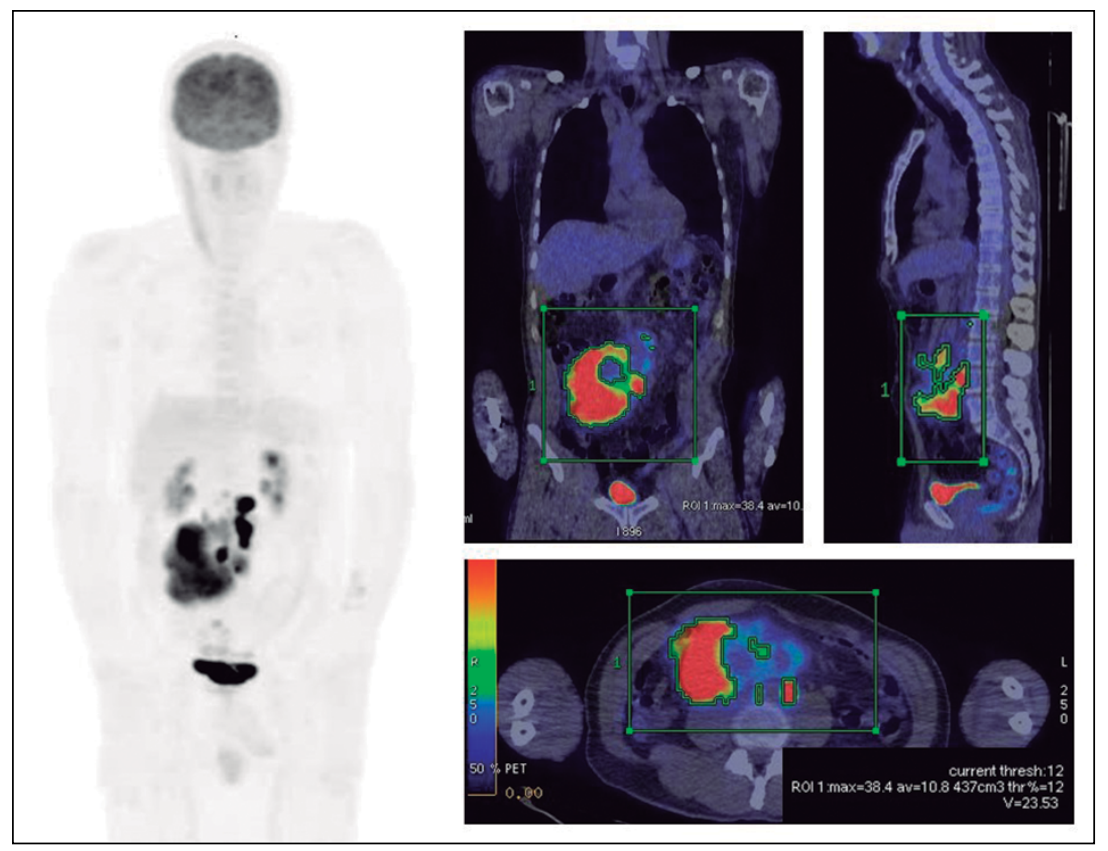

Figura 1. Cuantificación en $\mathrm{PET} / \mathrm{CT}$. El área de interés debe comprender completamente la lesión, excluyendo la captación fisiológica de estructuras adyacentes como el riñón y las vías urinarias. El Software proporciona el SUVmax: 38,4 , el SUVpromedio: 10,8, el volumen metabólico total: $437 \mathrm{cc}$. La masa tumoral glicolitica (MTG) corresponde al SUVpromedio $\mathrm{x}$ VMT: 4719,6. 


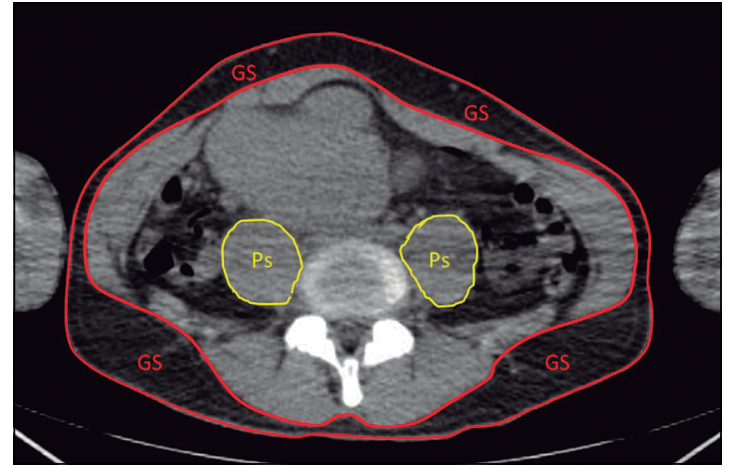

Figura 2. Cuantificación en la TC no contrastada en el mismo paciente de la Figura 1. El área de interés alrededor del psoas (Ps) entrega una medida de densidad (65 UH el derecho, 58 UH el izquierdo) y una medida de área $\left(17,5 \mathrm{~cm}^{2}\right.$ el derecho y $15,7 \mathrm{~cm}^{2}$ el izquierdo). El índice muscular del psoas (IMP) corresponde al promedio del área de ambos psoas dividido por la talla al cuadrado: $16,6 /(1,74 \mathrm{~m})^{2}=5,5 \mathrm{~cm}^{2} / \mathrm{m}^{2}$. El área de interés alrededor del tejido graso subcutáneo (GS) permite calcular el índice de grasa subcutánea (IGS) mediante la misma fórmula: $79,0 /(1,74 \mathrm{~m})^{2}=26,1 \mathrm{~cm}^{2} / \mathrm{m}^{2}$. de grasa subcutánea se midió de forma manual dibujando el perímetro del abdomen y perímetro de la pared muscular a nivel L3 (Figura 2), y se dividió por la estatura según la fórmula: IGS = $\mathrm{cm}^{2} / \mathrm{m}^{2}{ }^{17}$. Sus valores también fueron normalizados según sexo.

Para estimar la respuesta precoz a terapia mediante PET/CT interino se utilizó la escala Deauville (escala de 5 puntos o de Lugano), que es el criterio más utilizado en la actualidad para estimar respuesta precoz a terapia en linfomas ávidos de F18-FDG'. Un score 1, 2 o 3 fue considerado "respuesta completa" (Figura 3), y un score 4 o 5 "respuesta parcial" o "progresión" según correspondiera (Figura 4).

El estado vital de los pacientes se investigó recurriendo a la ficha clínica y al registro civil. Conociendo el estado vital, fecha de defunción en caso de muerte, y causa de fallecimiento, se clasificaron como fallecidos para efectos de determinación de supervivencia cáncer-específica, aquellos pacientes

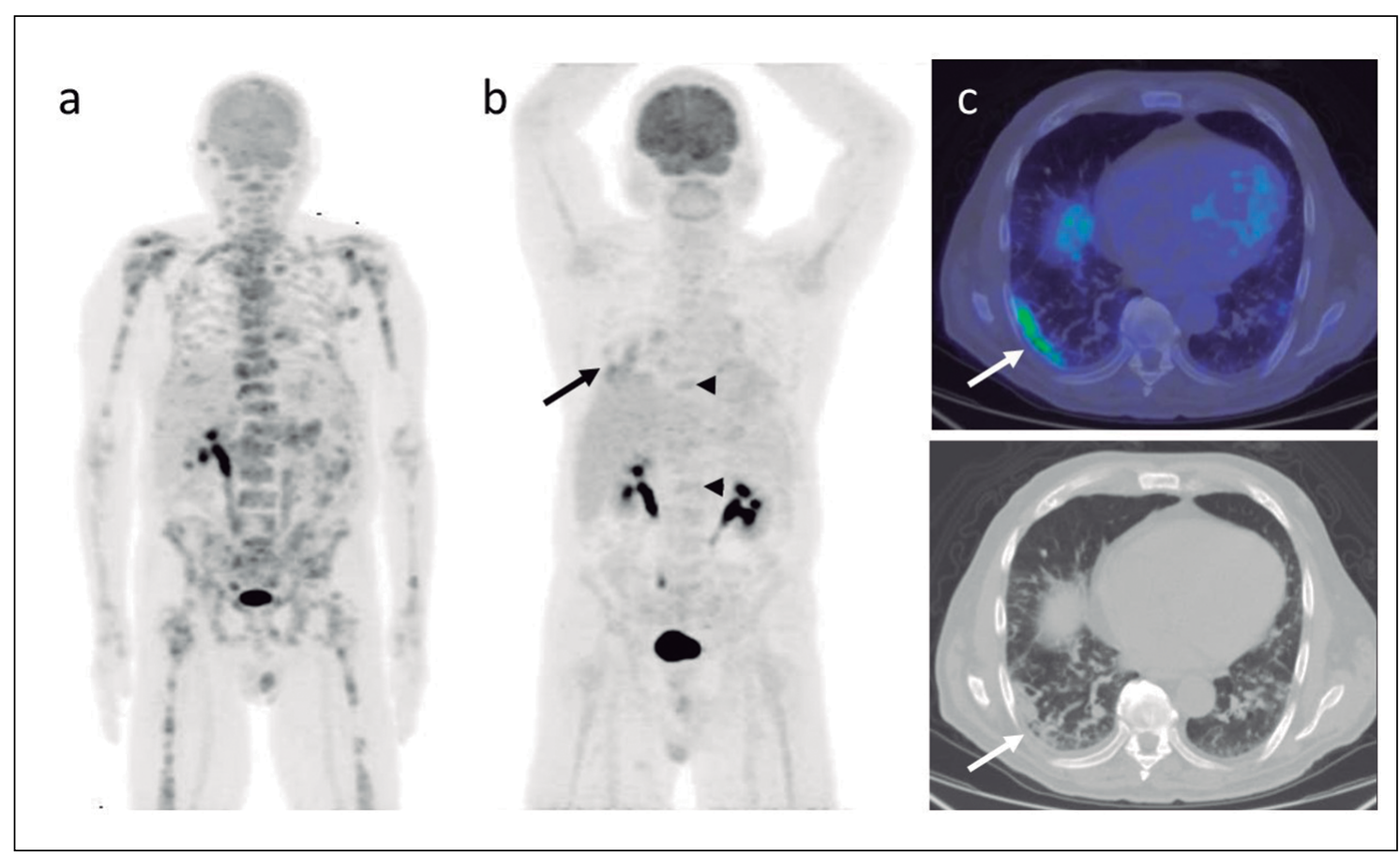

Figura 3. Hombre de 70 años con LNH doble expresor con buena respuesta a terapia. a) PET/CT de etapificación con extenso compromiso nodal y extranodal (Ann Arbor IV). b) PET/CT interino después del $2^{\circ}$ ciclo de quimioterapia muestra respuesta completa al tratamiento, con persistencia sólo de escasa captación vertebral (cabezas de flecha) similar a la actividad fisiológica del hígado, compatible con Score 3 de Deauville. Han aparecido focos hipercaptantes pulmonares de origen inflamatorio-infeccioso o neumonía en organización (flechas), que se muestran en cortes axiales en c). 


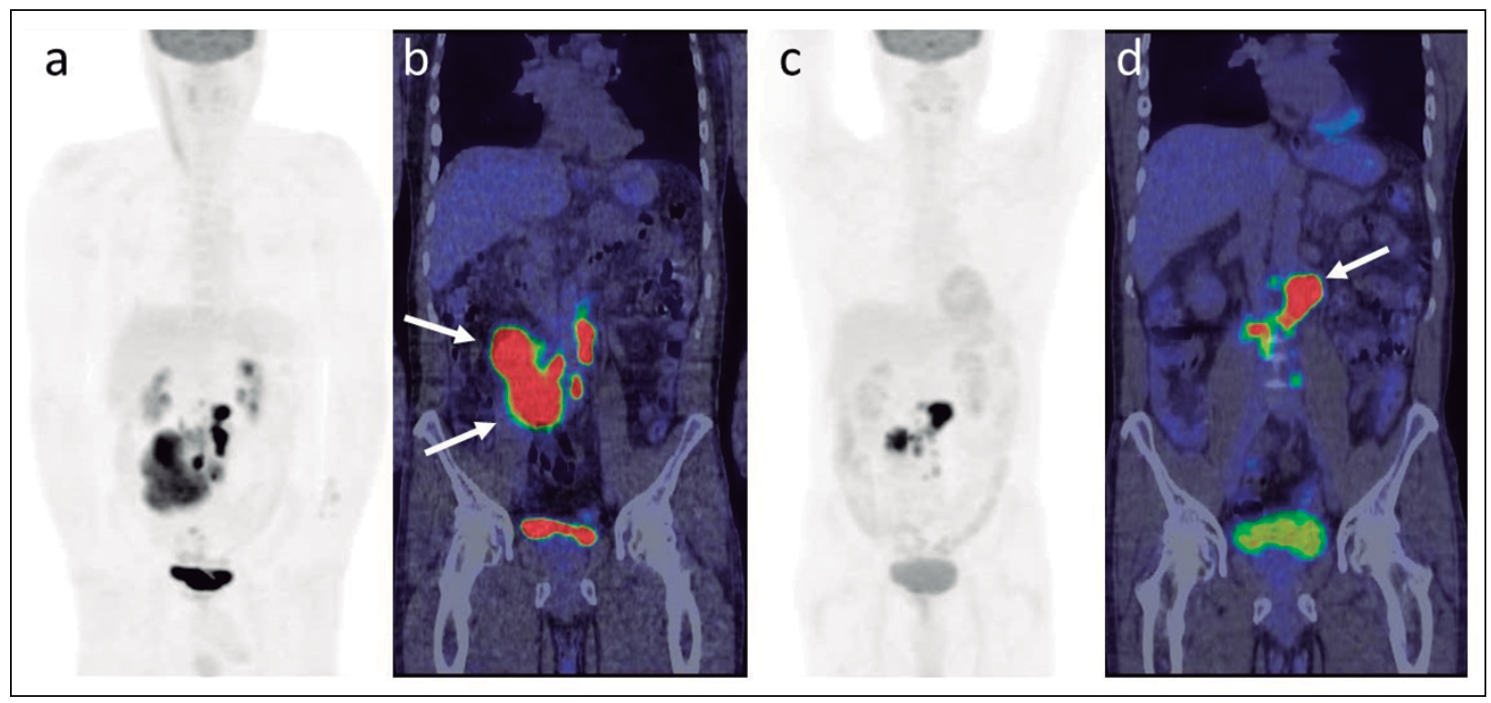

Figura 4. Hombre de 57 años con LNH DCG com mala respuesta a terapia. a y b) PET/CT de etapificación revela conglomerado de adenopatías mesentéricas y retroperitoneales (flechas), sin compromiso extranodal (Ann Arbor II). c y d) El PET/CT interino muestra respuesta parcial en adenopatías conocidas, y aparición de adenopatías retroperitoneales (flecha) compatible con progresión de la enfermedad (Deauville 5).

que presentaron "linfoma" como causa de muerte primaria o secundaria. Aquellos pacientes con fallecimiento de otra causa fueron considerados vivos hasta ese momento. Dichos pacientes y aquellos no fallecidos durante el tiempo de observación fueron considerados como "censurados" para los efectos del análisis de sobrevida.

\section{Estudio estadístico}

Se confeccionaron curvas de sobrevida de Kaplan-Meier, y se utilizó log-rank test y regresión multivariable de Cox para analizar el valor pronóstico de sobrevida de cada una de las variables. Para esto se utilizó programa MedCalc versión 18.10.2 (MedCalc Software Ltd., Seúl, República de Corea). Los mejores valores de corte para estimar sobrevida de las variables numéricas estudiadas se obtuvieron utilizando curvas ROC. Para estudio de correlación se utilizó Spearman rank test.

\section{Aspectos éticos}

La base de datos PET/CT de la cual fueron seleccionados los casos cuenta con la aprobación para su utilización con estos fines por el Comité de Ética de la institución. Todos los pacientes incluidos en este estudio han adherido y firmado un consentimiento informado para realizar inves- tigación utilizando los estudios de PET/CT. Para efectos del análisis de los datos y la publicación del estudio, se usó la información codificada. Se protegió la privacidad y confidencialidad de los pacientes del estudio.

\section{Resultados}

Se evaluó un total de 138 pacientes con $\mathrm{LNH}$ (mediana: 61 años, rango: 15-87 años, 60,4\% hombres), de los cuales 31 fallecieron por el LNH (mediana seguimiento: $39 \mathrm{~m}$, rango: 1-115 m). Los subtipos histológicos y mortalidad son mostrados en la Tabla 1. Todos los pacientes fueron tratados inicialmente con quimioterapia según protocolos internacionales, basados principalmente en esquemas CHOP o R-CHOP.

Las variables estudiadas se muestran en la Tabla 2. Los factores pronósticos más poderosos fueron presencia de síntomas B (p: 0,0007), nivel de hemoglobina sanguínea $(\mathrm{p}<0,0001)$, nivel de albúmina sanguínea (p: 0,001), área del psoas ( $\mathrm{p}<0,0001)$, IMP ( $\mathrm{p}<0,0001)$, tamaño de la lesión dominante (p:0,0024), VMT ( $<<0,0001)$, MTG $(\mathrm{p}<0,0001)$, y mala respuesta al PET/CT interino $(\mathrm{p}<0,0001)$. Los mejores valores de corte 
Tabla 1. Subtipo histológico y mortalidad específica de 138 pacientes con LNH

\begin{tabular}{|lrc|}
\hline Subtipo histológico & n & Fallecidos \\
\hline DCG & 48 & $9(19 \%)$ \\
Doble expresor & 6 & $2(33 \%)$ \\
\hline Folicular & 22 & $1(5 \%)$ \\
LLC & 10 & $2(20 \%)$ \\
\hline MALT & 8 & $2(25 \%)$ \\
Manto & 10 & $4(40 \%)$ \\
\hline Transformación & 6 & $1(17 \%)$ \\
Otros LNH tipo B & 16 & $5(31 \%)$ \\
\hline LNH tipo T & 11 & $5(45 \%)$ \\
\hline LNH Natural Killer & 1 & $0(0 \%)$ \\
\hline TOTAL & 138 & $31(22 \%)$ \\
\hline
\end{tabular}

LNH: Linfoma no Hodgkin; DCG: Difuso de células grandes; LLC: Leucemia linfática crónica; MALT: Mucosa-associated lymphoid tissue.

de las variables estudiadas se obtuvieron mediante curvas ROC, y se muestran en la Tabla 2.

El IMC no mostró valor pronóstico, sin embargo, presentó una alta correlación con el IGS (rho: 0,828, p < 0,0001), índice que sí exhibió utilidad pronóstica. La correlación entre el IMC y el IMP fue menor, aunque significativa (rho: 0,316, p: 0,0002).

En el análisis multivariable comparativo entre los factores más poderosos, el PET/CT interino fue la variable independiente de mayor significación (p: 0,0003).

Las curvas de Kaplan-Meier de los factores pronósticos más relevantes con sus valores de corte se muestran en la Figura 5.

\section{Discusión}

Los factores pronósticos en LNH permiten predecir la evolución de los pacientes, y estimar sobrevida ${ }^{9}$. Su sustento teórico se basa principalmente en dos conceptos: condición del paciente para enfrentar la patología, y avance de la enfermedad al momento del diagnóstico ${ }^{10}$. Ambos, aunque son teóricamente distintos, suelen ir de la mano de manera inversamente proporcional, ya que enfermedades avanzadas generalmente se asocian a pacientes más comprometidos, y pacientes en buenas condiciones generales comúnmente tienen enfermedades menos extensas. En nuestra casuística, tanto los factores propios del paciente (Hemoglobina, albuminemia, masa muscular y cantidad de tejido adiposo subcutáneo) como aquellos propios de la enfermedad (LDH, Ann Arbor, VMT o "carga tumoral", SUVmax) mostraron significativo valor pronóstico de sobrevida, lo que coincide con la literatura ${ }^{5,7,9}$.

El IPI es posiblemente la evaluación pronóstica más empleada en $\mathrm{LNH}^{9,26}$. Se basa en la edad, estimación clínica de la condición general del paciente ("performance status"), laboratorio (LDH), e imágenes. De estos factores, el nivel de LDH, el número de órganos extranodales comprometidos y la etapa Ann Arbor mostraron valor pronóstico en nuestro estudio, y sólo la edad no lo hizo. De los factores clínicos no considerados en el IPI, destacamos el elevado valor pronóstico de los síntomas B obtenido, constituyendo el factor clínico más relevante en este estudio.

La medición de proporción de masa muscular y de tejido adiposo al momento del diagnóstico, aparecen como factores de gran valor pronóstico en nuestra muestra, coincidiendo con la escasa literatura disponible ${ }^{17-19}$. La sarcopenia, y en menor grado la lipopenia, son considerados un factor de riesgo en pacientes oncológicos, y se han asociado a peor pronóstico ${ }^{25}$. Además de su evidente utilidad pronóstica, son de fácil disponibilidad, ya que se pueden obtener desde cualquier TC de abdomen, examen que por lo demás es ampliamente utilizado en patología oncológica. Nosotros utilizamos una simple medición del área del psoas y del celular subcutáneo abdominal a nivel L3, sin utilización de software específicos, demostrando utilidad pronóstica en nuestra serie, coincidiendo con la literatura ${ }^{19,23}$. Creemos que estas variables de composición corporal podrían ser de utilidad en la evaluación inicial de pacientes con $\mathrm{LNH}$, junto al IPI y otros factores pronósticos.

La respuesta a la terapia medida intratratamiento con el PET/CT interino, parece ser el único factor pronóstico independiente de las variables previamente descritas, ya que no depende del estado intrínseco del paciente, y tampoco necesariamente del grado de avance de la enfermedad. Posiblemente por este motivo, el análisis multivariable de Cox le asigna el mayor valor pronóstico de sobrevida. Esto es de gran importancia, por 
Tabla 2. Variables en estudio y su significación pronóstica utilizando regresión de Cox

\begin{tabular}{|c|c|c|c|c|c|}
\hline \multicolumn{3}{|l|}{ Variables cuantitativas } & \multicolumn{3}{|l|}{ Variables cualitativas } \\
\hline & $\mathbf{p}$ & n medible & & $\mathbf{p}$ & n medible \\
\hline Clínicas & & & Clínicas & & \\
\hline - Edad & NS & 138 & - Edad > 60 años & NS & 138 \\
\hline - Peso & NS & 138 & - Sexo & NS & 138 \\
\hline - Talla & NS & 137 & - Síntomas B & 0,0007 & 89 \\
\hline$-\mathrm{IMC}$ & NS & 137 & - Obesidad & NS & 137 \\
\hline Laboratorio & & & - IMC normal & NS & 137 \\
\hline - Albúmina & 0,001 & 95 & Laboratorio & & \\
\hline- LDH & 0,023 & 109 & - Albúmina $<4$ g/dl & 0,0010 & 95 \\
\hline - Hemoglobina & $<0,0001$ & 108 & $-\mathrm{LDH}>170$ & 0,0426 & 109 \\
\hline - Leucocitos & NS & 104 & - Hemoglobina $<12$ g/dl & $<0,0001$ & 108 \\
\hline - VHS & 0,0191 & 90 & - VHS > $20 \mathrm{~mm} / \mathrm{h}$ & 0,0483 & 90 \\
\hline - Beta-2-microglobulina & NS & 31 & Anatomía patológca & & \\
\hline & & & - Biopsia MO (+) & NS & 76 \\
\hline Anatomía patológca & & & - LNH B vs T & NS & 138 \\
\hline - Ki67 & NS & 55 & - Agresivo vs Indolente & 0,0055 & 138 \\
\hline Composición corporal & & & Composición corporal & & \\
\hline - Área psoas & $<0,0001$ & 138 & - Área psoas $<14 \mathrm{~cm}^{2}$ & 0,0005 & 138 \\
\hline - Densidad psoas & 0,0500 & 131 & - Densidad psoas < 56 UH & 0,0048 & 131 \\
\hline$-\mathrm{IMP}$ & $<0,0001$ & 137 & - $\mathrm{IMP}<3,8 \mathrm{~cm}^{2} / \mathrm{m}^{2}$ & 0,0002 & 137 \\
\hline- IGS & 0,0065 & 136 & $-\mathrm{IGS}<70 \mathrm{~cm}^{2} / \mathrm{m}^{2}$ & 0,0163 & 136 \\
\hline PET/CT etapificación & & & PET/CT etapificación & & \\
\hline - Ann Arbor & 0,0202 & 138 & - Ann Arbor III-IV & 0,0067 & 138 \\
\hline$-N^{\circ} G G$ & 0,0068 & 138 & $-G G>4$ & 0,007 & 138 \\
\hline - Tamaño lesión dominante & 0,0024 & 130 & - Bulky & 0,0146 & 137 \\
\hline - Nº́rganos EN & 0,0249 & 138 & - Compromiso EN & NS & 138 \\
\hline - SUVmax & NS & 135 & - SUVmax > 11 & 0,0258 & 135 \\
\hline - VMT & $<0,0001$ & 136 & - VMT > $150 \mathrm{cc}$ & 0,0001 & 136 \\
\hline - MTG & $<0,0001$ & 134 & - MTG > 700 & 0,0019 & 134 \\
\hline $\mathrm{PET} / \mathrm{CT}$ interino & & & PET/CT interino & & \\
\hline - Deauville & $<0,0001$ & 92 & - Deauville $\geq 4-5$ & $<0,0001$ & 92 \\
\hline
\end{tabular}

IMC: índice de masa corporal; LDH: láctico dehidrogenasa; VHS: velocidad de eritrosedimentación; IMP: índice de masa del psoas; IGS: índice de grasa subcutánea; GG: Grupos ganglionares comprometidos; MO: médula ósea; EN: extranodal.

cuanto significa que un paciente, aun cuando se presente con una enfermedad avanzada o se encuentre en condiciones generales no óptimas, puede tener una evolución favorable si responde precozmente a la terapia.
En nuestro estudio sólo se incluyó los PET/ CT interinos realizados post $2^{\circ}$ o $3^{\circ}$ ciclo de quimioterapia, ya que controles post tratamiento efectuados posteriormente al $4^{\circ}$ ciclo o en el fin de la terapia, no representan necesariamente una 

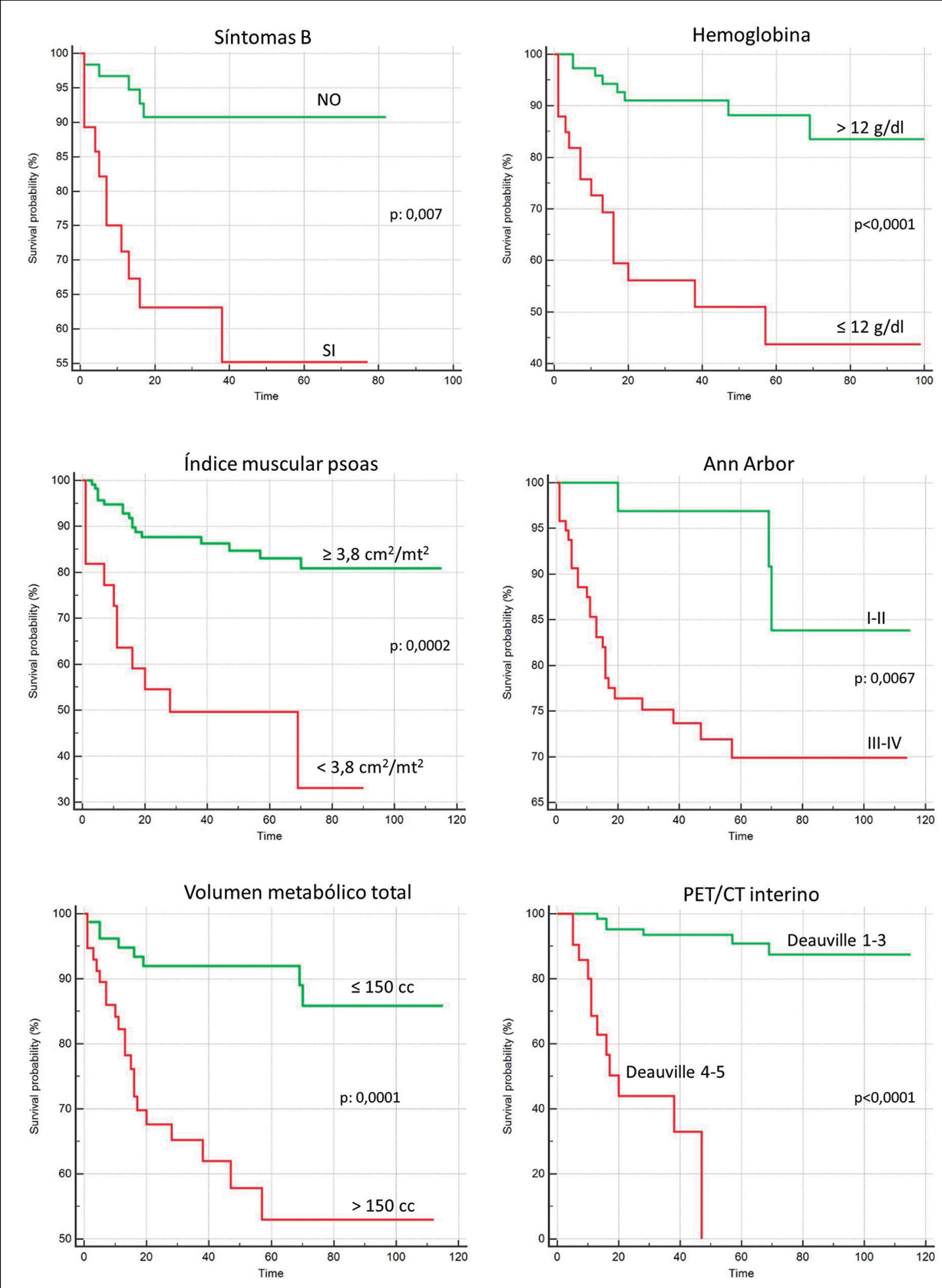

Figura 5. Curvas de sobrevida de Kaplan-Meier de los factores pronósticos más significativos. 
medición equivalente dado el proceso dinámico y progresivo de muerte de las células neoplásicas durante los ciclos consecutivos de quimioterapia. La pérdida de datos de estos pacientes, aunque marginal, representa una limitación de nuestro estudio.

Hoy se reconoce que el PET/CT interino, además de poseer valor pronóstico de sobrevida, es útil en el manejo del paciente, ayudando a reconocer precozmente a aquellos pacientes con enfermedad sensible a la terapia, y a identificar aquellos con mala respuesta, que podrían requerir modificaciones en sus esquemas terapéuticos ${ }^{2,9}$.

Sin embargo, la estimación de respuesta a la terapia mediante PET/CT interino está indicado sólo en aquellos linfomas ávidos de FDG ${ }^{9}$, por lo que no tendría aplicación en control de tratamiento de linfomas hipocaptantes, que corresponden generalmente a aquellos de bajo grado.

Dado la gran diversidad de subtipos de LNH, con amplia gama de comportamientos que van desde formas indolentes a cursos muy agresivos, es necesario efectuar estudios pronósticos específicos para cada uno de los subtipos histológicos de $\mathrm{LNH}$, materia que queda propuesta para futuros proyectos de investigación.

\section{Conclusión}

Existen diversos factores pronósticos útiles en $\mathrm{LNH}$, los que se basan principalmente en factores intrínsecos del paciente y en el grado de avance de la enfermedad al momento del diagnóstico. El $\mathrm{PET} / \mathrm{CT}$ interino mide la respuesta a terapia, y no está asociado directamente a dichos factores. En nuestra muestra el PET/CT interino resultó ser el factor pronóstico de sobrevida más poderoso en pacientes con $\mathrm{LNH}$.

\section{Referencias}

1. Guía Clínica AUGE LINFOMA en personas de 15 años y más. Serie Guías Clínicas MINSAL, 2013. [Internet]. Disponible en: http://www.bibliotecaminsal.cl/wp/ wp-content/uploads/2016/04/Linfoma-en-personas-de-15-a\%C3\%B1os-y-m\%C3\%A1s.pdf [Consultado el 11 de abril de 2020].

2. Shankland KR, Armitage JO, Hancock BW. Non-Hodgkin lymphoma. Lancet 2012; 380: 848-57.
3. National Cancer Institute. Surveillance, epidemiology and end results program (SEER). [Internet]. Disponible en: https://seer.cancer.gov/statfacts/html/nhl.html [Consultado el 8 de mayo de 2020].

4. Freedman A, Caron J, Mauch P, Aster J. Non Hodgkin's Lymphoma. En: DeVita V, Lawrence T, Rosenberg S, Ed. Lymphomas and Leukemias. China: Wolters Kluwer; 2016, 10th ed: 495-529.

5. Cancer.org. American Cancer Society. [Internet]. Disponible en: https://www.cancer.org/content/dam/ cancer-org/cancer-control/en/cancer-types/non-hodgkin-lymphoma-complete.pdf. [Consultado el 11 de abril de 2020].

6. Swerdlow SH, Campo E, Pileri SA, Harris NL, Stein H, Siebert R, et al. The 2016 revision of the World Health Organization classification of lymphoid neoplasms. Blood 2016; 127: 2375-90.

7. Undertanding non-Hodgkin Lymphoma. Lymphoma Research Foundation. Disponible en: https://ymphoma.org/wp-content/uploads/2018/03/LRF1607-NHLBooklet-D5V1-for-web-V2_3.9.2018.pdf. [Consultado el 16 de abril de 2020].

8. Casulo C, Burack WR, Friedberg JW. Transformed follicular non-Hodgkin lymphoma. Blood 2015; 125: 40-7.

9. National Comprehensive Cancer Network. NCCN Guidelines. Disponible en: http://www.nccn.org/professionals/physician_gls/f_guidelines.asp\#site. [Consultado el 16 de abril de 2020].

10. Nicolaides C, Dimou S, Pavlidis N. Prognostic Factors in Aggressive Non-Hodgkin's Lymphomas. The Oncologist 1998; 3: 189-97.

11. Bar-Shalom R, Yefremov N, Haim N, Dann EJ, Epelbaum R, Keidar Z, et al. Camera-based FDG PET and $67 \mathrm{Ga}$ SPECT in Evaluation of Lymphoma: Comparative Study. Radiology 2003; 227: 353-60.

12. Ceriani L, Martelli M, Zinzani PL, Ferreri AJM, Botto B, Stelitano C, et al. Utility of baseline 18FDG-PET/CT functional parameters in defining prognosis of primary mediastinal (thymic) large B-cell lymphoma. Blood 2015; 126: 950-6.

13. Bailly C, Carlier T, Berriolo-Riedinger A, Casasnovas O, Gyan E, Meignan M, et al. Prognostic Value Of FDGPET In Patients With Mantle Cell Lymphoma: Results From The LyMa-PET Project. Haematologica 2020; 105: e33-6; Doi: 10.3324/haematol.2019.223016.

14. Wang H, Shen G, Jiang C, Li L, Cui F, Tian R. Prognostic value of baseline, interim and end of-treatment 18F-FDG PET/CT parameters in extranodal natural killer/T-cell lymphoma: A meta-analysis. PLoS ONE 13 (3): e0194435. https://doi.org/10.1371/journal. pone. 0194435 . 
15. Weiler-Sagie M, Bushelev O, Epelbaum R, Dann EJ, Haim N, Avivi I, et al. 18F-FDG Avidity in Lymphoma Readdressed: A Study of 766 Patients. J Nucl Med 2010; 51: $25-30$.

16. Ladrón de Guevara D, Briceño G, Cid S, Durán F, Itriago L, Álvarez M. Compromiso nodal y extranodal detectado con PET/CT en linfoma en etapificación. Rev Chil Radiol 2017; 23: 91-7.

17. Camus V, Lanic H, Kraut J, Modzelewski R, Clatot F, Picquenot JM, et al. Prognostic impact of fat tissue loss and cachexia assessed by computed tomography scan in elderly patients with diffuse large B-cell lymphoma treated with immunochemotherapy. Eur J Haematol 2014; 93: 9-18.

18. Go S, Park MJ, Song HN, Kim HG, Kang MH, Lee HR. Prognostic impact of sarcopenia in patients with diffuse large B-cell lymphoma treated with rituximab plus cyclophosphamide, doxorubicin, vincristine, and prednisone. J Cachexia Sarcopenia Muscle 2016; 7: 56776.

19. Lanic H, Kraut-Tauzia J, Modzelewski R, Clatot F, Mareschal S, Picquenot JM, et al. Sarcopenia is an independent prognostic factor in elderly patients with diffuse large B-cell lymphoma treated with immunochemotherapy. Leuk Lymphoma 2014; 55: 817-23.

20. Cheson BD. PET/CT in Lymphoma: Current Overview and Future Directions. Semin Nucl Med 2017; 48: 7681.

21. Adams MC, Turkington TG, Wilson JM, Wong TZ. A Systematic Review of the Factors Affecting Accuracy of SUV Measurements. AJR 2010; 195: 310-20.

22. Lin EC, Alavi A, Kinahan P. Standarized uptake value. En: Lin EC, Alavi A. PET and PET/CT: A clinical guide. New York, USA: Thieme Medical Publishers Inc; 2005: 28-31.

23. Hamaguchi Y, Kaido T, Okumura S, Kobayashi A, Hammad A, Tamai Y, et al. Proposal for new diagnostic criteria for low skeletal muscle mass based on computed tomography imaging in Asian adults. Nutrition 2016; 32: 1200-5.

24. Albano D, Messina C, Vitale J, Sconfienza LM. Imaging of sarcopenia: old evidence and new insights. European Radiology 2020; 30: 2199-208.

25. Derstine BA, Holcombe SA, Ross BE, Wang NC, Su GL, Wang SC. Skeletal muscle cutoff values for sarcopenia diagnosis using T10 to L5 measurements in a healthy US population. ScientIfIc Reports 2018; 8: 11369. DOI: 10.1038/s41598-018-29825-5.

26. Guías Prácticas Clínicas para el Diagnóstico y Tratamiento de Linfomas No Hodgkin. [Internet]. Disponible en: https://www.sochihem.cl/site/pagina.php?id=183 [Consultado el 11 de abril de 2020]. 